\title{
"Project Expose"- Radiotherapy Advocacy in the Hospital - Nurses Do It Best!
}

\author{
Christine Dominguez, Amanda Marriott, Gerald B Fogarty* \\ Department of Radiotherapy, Genesis Care, St Vincent's Hospital, Sydney, Australia \\ *Corresponding Author: Dr. Gerald B Fogarty, Department of Radiotherapy, Genesis Care, St Vincent's Clinic, \\ 438 Victoria St, Darlinghurst, Sydney, Australia, Tel: +61-2-83025400; E-mail: Gerald.fogarty@genesiscare.com
}

Received: 08 October 2018; Accepted: 16 October 2018; Published: 22 October 2018

\begin{abstract}
Introduction: Better radiotherapy (RT) modalities and techniques with efficacy proven in prospective trials have led to an increase the indications for RT. In our own large Australian metropolitan tertiary referral hospital however, general staff and students have little appreciation of this. Advocacy is needed for this effective intervention to become more known and accessible.
\end{abstract}

Method: We launched "Project Expose", an educational intervention aimed at the general hospital staff. The main component was a guided tour of our department where all radiation craft groups explained what they do, while showing how the radiation machines function. We wanted to measure the outcome of this intervention.

Results: 500 were invited, 350 invitations were distributed, 45 attended (45/350 - 13\%). Of these, 10 responded to an invitation that accompanied a purpose designed business card, 8 were by word of mouth from those who had previously attended. 14 had been encouraged by an educator to attend. 32/45 (71\%) completed the post tour survey. Of these, 11 were nurses, 9 were allied health, 7 were health care students, 4 were non-health occupation or students, and 1 was medical staff. 97\% of survey respondents said their knowledge about modern radiotherapy had increased.

Discussion: The tour was not a success in that it was difficult to attract general hospital staff (13\%). The intervention was a success for those that attended, with $97 \%$ of survey completers said they had increased in knowledge of modern radiotherapy. An important finding was the pivotal a role of nurses in radiotherapy advocacy. 
Keywords: Radiotherapy; Patient advocacy; Nursing; Education; Culture; Australia

\section{Introduction}

Better radiotherapy (RT) modalities and techniques, with efficacy proven in prospective trials, have led to an increase in the indications [1]. Patient and health-care worker driven advocacy is needed for patients to be referred for these new treatments. In the Australian community, however, there is misinformation about RT that prevents this advocacy getting traction [2]. To address this, there has been some effective leadership in this area by the Faculty of Radiation Oncology, of the Royal Australian and New Zealand College of Radiologists [3]. In our own large Australian metropolitan tertiary referral hospital however, our impression was that, in general, health care staff and students have little appreciation of RT, especially in how it has improved in the last few years. We decided to take responsibility for increasing awareness and to develop strategies to make RT better known. At a department staff meeting we decided to launch "Project Expose", an educational intervention aimed at the general hospital staff. The main component was to be a talk by our medical director and then a guided tour of our department where all radiation craft groups were to explain what they do, while showing how the radiation machines function. This was followed by a session where questions could be asked, and feedback obtained.

\section{Methods}

A radiation staff member lead was sourced to act as the lead person. This person was to be the main organizer, the point of contact, the tour guide and the person that distributed and collated a post-tour survey. A one-hour education session was devised. The whole educational intervention involved a 15 -minute talk by the medical director of the department about modern RT, then the lead person would take the tour group to different parts of the department including nursing, planning, treatment and physics. A free morning tea was then provided with the director in attendance to field any questions and suggestions. The tours were had regularly on a weekly basis at a time we fixed as a department. A business card to make the invitations easier was produced. A dedicated email address was created. A survey was constructed (Supplementary figure 1) with sentences asking about the background of the person, for example, their role in the hospital, how they had been invited to the tour. They were asked also about how the tour had impacted them and about how the tour could be improved, mentioning different parts of the tour so that that would be able to refresh their memory. The lead person would ask the tour participants during the morning tea time to complete the survey. The surveys were collected, collated, findings were to be summarized and conclusions presented. Our ethics review staff decided an ethics submission was not needed as the intervention was an education session for staff who volunteered, there were no patients involved and there were no problems with confidentiality of patient treatment.

\section{Results}

\subsection{Sourcing a staff lead}

Initially we thought the natural lead for this project would be a radiation therapist, who is the most common staff members in a radiation facility. They have excellent communications with all the radiation craft groups within the 
department. However, there were no volunteers despite group enthusiasm and personal encouragement. This delayed the project for months. Other craft groups were then approached, and the radiation nurses eagerly took up this challenge and were proactive in making the project come alive.

\subsection{Invitations}

3.2.1 Business cards: 500 were produced. In a 6-month period, 350 were distributed by those on the staff that interacted with the general staff of the hospital. The card distributors included the doctors, the practice manager, the nurses etc. When explained to a potential attendee, there was never any negativity to the tour idea. All were interested and delighted to be asked. Most promised to come at some stage.

3.2.2 More audacious ways to invite: When it was clear that people were not coming, more audacious ways of attracting them were tried e.g. our nurse lead would sit with students during the lunch breaks in the general cafeteria and hospital gardens to ask them directly. The medical director approached different educators around the hospital.

3.2.3 Overall: In our estimation over 500 individuals were asked. 350 business cards were distributed but only 45 attended (13\%). 32 of $45(71 \%)$ completed the post tour survey. These were mostly those who stayed for morning tea. Of those that attended and filled the survey, 10 responded to an invitation that accompanied a purpose-designed business card, 8 were by word of mouth from those who had previously attended a tour, 14 had been encouraged by an educator to come along. The most effective and efficient ways to get tour participants were always through the nursing contacts. As many had booked in by email, we asked those who attended but did not fill the survey to complete the survey via email. One of 13 did this.

\subsection{Surveys}

Of those that completed the survey, 11 were nurses, 9 were allied health, 7 were health care students, 4 were nonhealth occupation or students and 1 was medical staff. Thirty-one out of the 32 (97\%) who responded thought the tours were excellent and acknowledged that, by attending the tour, they had learnt about modern radiotherapy (Supplementary Figure 2). One suggested that the role of radiation nursing could be better explained. Another suggested that more explanation of the technical side of radiotherapy could be beneficial. Five wanted to have a longer tour.

\section{Discussion}

This small study looked at the effectiveness of an educational intervention that included a tour of the radiation oncology department in raising awareness of modern RT. 500 individuals were asked. 350 business cards were distributed but only 45 attended (13\%). 32 of 45 (71\%) completed the post tour survey. 31 of 32 (97\%) of those who filled out the post tour survey thought that by attending, they had learnt about modern radiotherapy. There were many other learnings and flow-on from this exercise. It was difficult to get a radiation therapy lead. In hindsight, the reason is obvious. Radiation therapists are the most common person in the department but absent outside the 
department in the general hospital. The nurses were able to effectively communicate, mainly through their nursing colleagues outside the department, but still within the hospital. The reason in hindsight is also clear. Nurses are the most ubiquitous health care professionals in the hospital and play a significant part in harmonious interdepartmental communication. The radiation nurses used every opportunity to promote this tour amongst general staff in the hospital, particularly their own nursing colleagues in other wards.

Many hospital staffs were asked, all were enthusiastic, but few came. The reason for this may have been the timing. We picked a time that was convenient for our department, 9-10 am on a Thursday morning, when our staff were available. However, we learnt that this was not convenient for the general hospital staff. Outpatient nurses wanted 89 am prior to clinics starting; ward nurses wanted 2.30-3.30 pm, around handover time. We compromised by saying that we would do a tour at any time provided there were at least four people. By doing this we were able to get all the chemotherapy nurses (ten) through in two lots over two weeks. Still, the tours were difficult to get people to attend despite the enthusiasm. Nurses were the most common tourist. This maybe because nurses feel keenly their duty as patient advocates [4]. Often the nurse, especially the oncology nurse, is the person who is asked about radiotherapy by the patient who is waiting for radiotherapy. Nurses want to be ready for this [5]. Of those that did attend, there was a reasonable survey response, 32 of $45(71 \%)$. Of those that did respond they were almost unanimous (31 of 32-97\%) in thinking the tours worthwhile and helped them to increase in their knowledge of modern radiotherapy.

There were further corollaries from this study. What was pleasantly surprising was the participants were quite fascinated by the improvement in radiotherapy modalities and techniques, and this increased the radiation staff confidence. It was also quite pleasing to see how the other radiation craft groups responded well to the invitation of the nurse lead to explain themselves to the tour groups, and this certainly helped with inter-craft group culture within the department. The tours led to a ripple effect through the campus that benefitted our centre. The general staff were more aware that the Radiation Oncology department existed and that we are happy to have people in our department. Other departments in the hospital realized that radiotherapy wanted to be known and some educators and directors encouraged their staff to come. Another department wants to conduct their own tours to become better known, but this has yet to happen. A major discovery was that this educational event demonstrated the importance of radiation nursing to radiation advocacy in the hospital. The nurse lead was pivotal in making the tours happen. Nurses were the most common persons to do the tours. Nurses were the ones who most encouraged others to come. This study emphasized that nurses are important for radiation advocacy both for patients and for our hospital. Nurses do it best!

\section{Conclusions}

Educational initiatives including tours of the RT department are well received by the general hospital staff. Those who attend learn about modern radiotherapy. Getting staff to attend is difficult. Nurses were the best at promotion and attending the initiative. Radiation oncology administrators trying to promote modern radiotherapy should consider that, based on this study, centers can promote and attract general hospital staff to the department for 
education about modern radiotherapy. They should also acknowledge that nurses can play an important role in radiotherapy advocacy among hospital staff.

\section{Funding Details}

There was no specific funding.

\section{Acknowledgements}

We would like to acknowledge the support of Genesis Cancer Care in fulfilling this project. This project was presented at a Genesis Cancer Care collaboration conference in Perth in 2017 and was well received by the radiation multidisciplinary community.

\section{Conflict of Interests}

Authors declare that there is no conflict of interest.

\section{References}

1. Pfeffer MR, Blumenfeld P. The Changing Paradigm of Radiotherapy in the Elderly Population. Cancer J. 23 (2017): 223-230.

2. Sundaresan P, King M, Stockler M, et al. Barriers to radiotherapy utilization: Consumer perceptions of issues influencing radiotherapy-related decisions. Asia Pac J Clin Oncol 13 (2017): e489-e496.

3. Targeting Cancer, Faculty of Radiation Oncology. The Royal Australian and New Zealand College of Radiologists. (https://www.targetingcancer.com.au/) (2018).

4. Tariman JD, Szubski KL. The evolving role of the nurse during the cancer treatment decision-making process: a literature review. Clin J Oncol Nurs 19 (2015): 548-556.

5. Wilkinson K. Informed consent and patients with cancer: role of the nurse as advocate. Clin J Oncol Nurs 16 (2012): 348-350.

Citation: Christine Dominguez, Amanda Marriott, Gerald B Fogarty. "Project Expose"- Radiotherapy Advocacy in the Hospital - Nurses Do It Best!. Archives of Clinical and Medical Case Reports 2 (2018): 135139.

\begin{tabular}{|c|c|}
\hline (c) (9) & $\begin{array}{l}\text { This article is an open access article distributed under the terms and conditions of the } \\
\text { Creative Commons Attribution (CC-BY) license } 4.0\end{array}$ \\
\hline
\end{tabular}

